\title{
ON MAXIMAL ABELIAN SUBRINGS OF FACTORS OF TYPE II $_{1}$
}

\author{
LAJOS PUKÁNSZKY
}

1. Introduction. Although we possess a fairly complete knowledge of the abelian subrings of rings of operators in a Hilbert space which are algebraically isomorphic to the ring of all bounded operators of a finite or infinite dimensional unitary space, that is of factors of Type I, very little is known of abelian subrings of factors ${ }^{1}$ of Type $\mathrm{II}_{1}{ }^{1}$. In (1), Dixmier investigated several properties of maximal abelian subrings of factors of Type II. It turned out that their structure differs essentially from that of maximal abelian subrings of factors of Type I. He showed the existence of maximal abelian subrings in approximately finite factors, ${ }^{2}$ possessing the property that every inner*-automorphism carrying this subring into itself is necessarily implemented by a unitary operator of this subring. These maximal abelian subrings are called singular. In addition, he constructed a $\mathrm{II}_{1}$ factor containing two singular abelian subrings which cannot be connected by an inner *-automorphism of this ring. ${ }^{3}$

The purpose of the present paper is to introduce new invariants for abelian subrings of a factor of Type $\mathrm{II}_{1}$. By means of these we shall be able to show the existence of infinitely many singular maximal abelian subrings of a factor of approximately finite type which, pairwise, cannot be connected by *automorphisms of this ring.

Indeed (cf. Lemma 1 below), we associate with every abelian subring of a $\mathrm{II}_{1}$ factor an abelian ring, such that the rings corresponding to abelian subrings connected by *automorphisms are unitarily equivalent. Since the spatial invariants of abelian rings in a Hilbert space are well known (4), we obtain a useful set of invariants for the abelian subrings, with the aid of which we construct various examples.

The author is indebted to the referee for his valuable criticism; in particular Lemma 5 in its present form was suggested by him.

Received January 8, 1959.

${ }^{1}$ We recall that a weakly closed self-adjoint operator algebra in a Hilbert space, which contains the unit operator (that is, a ring of operators), is a factor if its center consists only of the scalar multiples of the unit operator. A factor which is not of Type $I$ is of Type II $_{1}$ if all isometries contained in it are unitary transformations. For a theory of factors cf. (2). When speaking simply of a ring, we always mean a ring of operators in a Hilbert space.

${ }^{2} \mathrm{~A} \mathrm{II}_{1}$ factor is of approximately finite type, if it is generated by an ascending sequence of subfactors algebraically isomorphic to the full rings of finite dimensional unitary spaces. Two $\mathrm{II}_{1}$ factors of approximately finite type are algebraically isomorphic and every $\mathrm{II}_{1}$ factor contains such a subfactor. For details cf. (3).

${ }^{3} \mathrm{Cf}$. (1). This factor is very probably not of approximately finite type. 
2. The invariants. Let $\mathbf{M}$ be a $\mathrm{II}_{1}$ factor and $\mathbf{P}$ a maximal abelian subring of it. Let $\operatorname{Tr}(A) \quad(A \in \mathbf{M})$ be the canonical trace on $\mathbf{M}$. Putting $(X, Y)=\operatorname{Tr}\left(X Y^{*}\right)$ for $X, Y \in \mathbf{M}, \mathbf{M}$ becomes a pre-Hilbert space; let $\bar{H}$ be the completion of $\mathbf{M}$. If $A \in \mathbf{M}$, there exist two bounded operators $L_{A}$ and $R_{A}$ on $\bar{H}$, such that for $X \in \mathbf{M}$ we have $L_{A} X=A X$ and $R_{A} X=X A$ respectively. Let us denote by $\mathbf{R}(\mathbf{P})$ the weakly closed abelian ring in $\bar{H}$ generated by the sets of operators $\left\{L_{A} ; A \in P\right\}$ and $\left\{R_{A} ; .1 \in P\right\}$.

Lemma 1. Let $\mathbf{M}_{1}$ and $\mathbf{M}_{2}$ be two $\mathrm{II}_{1}$ factors, $\mathbf{P}_{1} \subset \mathbf{M}_{1}$ and $\mathbf{P}_{2} \subset \mathbf{M}_{2}$ two maximal abelian subrings. Let $\phi$ be $a{ }^{*}$-isomorphic mapping from $\mathbf{M}_{1}$ onto $\mathbf{M}_{2}$, which carries $\mathbf{P}_{1}$ onto $\mathbf{P}_{2}$. Then there exists a unitary mapping of the space $\bar{H}_{1}$ onto the space $\bar{H}_{2}$, which carries $\mathbf{R}\left(\mathbf{P}_{1}\right)$ onto $\mathbf{R}\left(\mathbf{P}_{2}\right)$.

Proof. For this it is enough to show the existence of a unitary mapping $U$ from $\bar{H}_{1}$ onto $\bar{H}_{2}$, such that

$$
U L_{A} U^{-1}=L_{\phi(A)}
$$

and

$$
U R_{B} U^{-1}=R_{\phi(B)}
$$

for $A, B \in \mathbf{M}$. By the uniqueness of the normalized traces in $I_{1}$ factors we have $\operatorname{Tr}_{1}(A)=\operatorname{Tr}_{2}(\phi(A))$, so that putting $U(X)=\phi(X)\left(X \in \mathbf{M}_{1}\right)$ we get an isometry between the dense linear manifolds $\mathbf{M}_{1} \in \bar{H}_{1}$ and $\mathbf{M}_{2} \in \bar{H}_{2}$, which can be extended to a unitary mapping $U$ from $\bar{H}_{1}$ onto $\bar{H}_{2}$. If $A \in \mathbf{M}_{1}, X \in \mathbf{M}_{2}$, then

$U L_{A} U^{-1} X=U L_{A} \phi^{-1}(X)=U\left(A \phi^{-1}(X)\right)=\phi\left(A \phi^{-1}(X)\right)=\phi(A) X=L_{\phi(A)} X$, and similarly

$$
U R_{A} U^{-1} X=R_{\phi(A)} X
$$

which proves our lemma.

As a consequence of Lemma 1 we may conclude that to all spatial invariants of the ring $\mathbf{R}(\mathbf{P})$ correspond properties of $\mathbf{P}$ which are invariant under ${ }^{*}$-isomorphisms of the ring $\mathbf{M}$ which contains $\mathbf{P}$. Now let $\mathbf{P}$ be an arbitrary abelian ring in the Hilbert space $H$. As is known (4), there exists a uniquely determined sequence of mutually orthogonal projections $P_{n} \in \mathbf{P}(n=1,2, \ldots,+\infty)$ the sum of which equals unity, such that the restriction of $\mathbf{P}$ into the subspace $P_{n} H$ is an $n$-fold copy of a maximal abelian ring. In particular, if $\mathbf{P}$ is unitarily equivalent to $\mathbf{P}_{1}$, then these sequences of projections in these two rings must correspond to each other. Our next objective is to construct a sequence $\mathbf{P}_{n} \quad(n=1,2, \ldots$,$) of singular maximal abelian subrings (cf. \S 1$ ) of an approximately finite $\mathrm{II}_{1}$ factor so that for $\mathbf{R}\left(\mathbf{P}_{n}\right)$ only $P_{1}$ and $P_{n}$ differ from zero. In this case clearly $\mathbf{R}\left(\mathbf{P}_{n}\right)$ and $\mathbf{R}\left(\mathbf{P}_{m}\right)$ cannot be unitarily equivalent for $n \neq m$, and so (Lemma 1) $\mathbf{P}_{n}$ and $\mathbf{P}_{m}$ cannot be connected by a *automorphism of $\mathbf{M}$. 
3. Examples. First we recall the following facts concerning the construction of factors of Type $\mathrm{II}_{1}(\mathrm{cf} . \mathbf{1} ; \mathbf{2} ; \mathbf{3})$. Let $G$ be a countably infinite group, and let $L^{2}(G)$ be the Hilbert space of all complex-valued square summable functions on $G$. For $a \in G$ and $f(x) \in L^{2}(g)$ define the unitary operator $U_{a}$ by

$$
\left(U_{a} f\right)(x)=f\left(a^{-1} x\right)
$$

and $V_{a}$ by

$$
\left(V_{a} f\right)(x)=f(x a) .
$$

Let $\mathbf{M}(G)$ be the operator ring generated by the set $\left\{U_{a} ; a \in G\right\}$. Then $\mathbf{M}(G)$ is a factor of Type $\mathrm{II}_{1}$ if and only if every non-trivial class of conjugate elements in $G$ is infinite. If, in addition, $G$ is the union of an increasing sequence of finite subgroups, then $\mathbf{M}(G)$ is approximately finite. ${ }^{4}$ Let $G_{0} \subset G$ be an abelian subgroup and denote by $\mathbf{P}\left(G_{0}\right)$ the abelian ring generated by the set $\left\{U_{a} ; a \in G_{0}\right\}$. Then $\mathbf{P}\left(G_{0}\right)$ is maximal if and only if for $a \bar{\epsilon} G_{0}$ the set $\left\{\mathrm{gag}^{-1} ; g \in G_{0}\right\}$ is infinite. $\mathbf{P}\left(G_{0}\right)$ is singular if $G$ has the following property: for every element $a \bar{\epsilon} G_{0}$ and arbitrary finite subset $B \subset G$ there exists an element $g_{0} \in G_{0}$, such that $a g_{0} a^{-1} \bar{\epsilon} G_{0}$ and from $g_{0} h^{-1}=g_{0}(g, h \in B)$ it follows that $g=h$.

Alternatively, the $\operatorname{ring} \mathbf{M}(G)$ can be described as follows. For $f, g \in L^{2}(G)$ define

$$
(f \times g)(x)=\sum_{y \in G} f\left(x y^{-1}\right) g(y)
$$

and $U_{f} g=f \times g$. Then $\mathbf{M}(G)$ is the collection of those operators $U_{f}$, for which $U_{f} g \in L^{2}(G)$ for every $g \in L^{2}(G)$. For such an $U_{f}$ its adjoint $U_{f}^{*}$ is $U_{\tilde{f}}$, where

$$
\tilde{f}(x)=\overline{f\left(x^{-1}\right)}
$$

and its trace is the value of the function $f(x)$ on the unit element of $G$, or $\operatorname{Tr}\left(U_{f}\right)=f(e)$. Let $G_{0} \subset G$ be an abelian subgroup and let us determine $\mathbf{R}\left(\mathbf{P}\left(G_{0}\right)\right)$. If $A=U_{f}$ and $B=U_{g}$ then

$$
\operatorname{Tr}\left(A B^{*}\right)=\operatorname{Tr}\left(U_{f} U_{g}^{*}\right)=\operatorname{Tr}\left(U_{f \times g}\right)=(f \times g)(e)=\sum_{x \in G} f(x) \overline{g(x)} .
$$

Since the set of elements in $L^{2}(G)$ for which $U_{f}$ is a bounded operator is dense in $L^{2}(G), \bar{H}$ (cf. Lemma 1) and $L^{2}(G)$ can be identified so that for

$$
A=U_{a}(a \in G) \quad\left(L_{A}\right) f(x)=f\left(a^{-1} x\right)
$$

and $\left(R_{A} f(x)=f(x a)(x \in G)\right.$. So finally $\mathbf{R}\left(\mathbf{P}\left(G_{0}\right)\right)$ can be identified with the ring in $L^{2}(G)$ generated by the set of operators

$$
\left\{U_{a}, V_{b} ; a, b \in G_{0}\right\} .
$$

${ }^{4}$ See footnote 2 . 
In the following, $G$ will denote a subgroup of the affine group over a countably infinite field $K$, obtained by restricting the subgroup of dilatations to an infinite subgroup $G_{0}$ of the multiplicative group of non-zero elements of $K{ }^{5} \mathrm{We}$ shall specify $K$ and this subgroup later in a way which is suitable for our purposes. Alternatively, in each case $G$ can be described as the set of all pairs $(a, \alpha)\left(a \in G_{0}, \alpha \in K^{+}\right.$, where we denote by $K^{+}$the additive group of $K)$, multiplication being defined as $(a, \alpha)(b, \beta)=(a b, a \beta+\alpha)$.

LEMma 2. Suppose that $K$ is the union of an increasing sequence of finite subfields. Then $\mathbf{M}(G)$ is a $\mathrm{II}_{1}$ factor of approximately finite type.

Proof. (1, p. 282). Observe first, that in this case $G$ is the union of an ascending sequence of finite subgroups. Therefore we have only to prove that every non-trivial class of conjugate elements in $G$ is infinite. Suppose first that $g=(a, \alpha)$, where $\alpha \neq 0$. Then $(c, 0)(a, \alpha)(c, 0)^{-1}=(a, c \alpha)$ and if $c$ runs over the elements of $G_{0}$ we get infinitely many elements. On the other hand, we have $(1, \gamma)(a, 0)(1, \gamma)^{-1}=(a, \gamma-a \gamma)$, therefore, if $a \neq 1$, we get again infinitely many different elements when $c$ varies over $K$, which proves our lemma.

It is of some interest to remark that Lemma 2 holds true even if $K$ is an arbitrary countable abelian field, though the proof for it is somewhat complicated. ${ }^{6}$

Lemma $2^{\prime}$. Let $K$ be an arbitrary countably infinite field. Then $\mathbf{M}(G)$ is a $\mathrm{II}_{1}$ factor of approximately finite type.

Proof. (For the following reasoning cf. (3, p. 793, § 5.5)). By the definition of the group $G$ the space $L^{2}(G)$ is the collection of all complex-valued square summable functions of the variables $a \in G_{0}$ and $\alpha \in K^{+}$. Let $X$ be the character group of $K^{+}$, and $\mu$ the normalized Haar measure on it, and $L^{2}(X)$ the Hilbert space of square integrable functions on it. For $f(a, \alpha) \in L^{2}(G)$ we denote by $F(a, \chi)(\chi \in X)$ the function on $G_{0} \times X$ obtained by taking the Fourier transforms of the functions $f(a, \alpha)$ for each fixed $a \in G_{0}$. Since

$$
\sum_{g \in G}|f(g)|^{2}=\sum_{a \in G_{0}} \int_{X}|F(a, \chi)|^{2} d \mu
$$

the correspondence $f \rightarrow F$ gives a unitary mapping from $L^{2}(G)$ onto $L^{2}\left(G_{0}\right) \cdot \otimes L^{2}(X)=H$. For $a \in G_{0}$ let us put $\chi^{a}(\alpha)=\chi(a \alpha)\left(\alpha \in K^{+}\right)$; it is well known that the collection of the mappings $\chi \rightarrow \chi^{a}$ is a representation of $G_{0}$ by automorphisms of the topological group $X$ which leaves the Haar measure invariant. Moreover, since for $\alpha \neq 0$ the set $\left\{a \alpha ; a \in G_{0}\right\}$ is infinite,

\footnotetext{
${ }^{5}$ The subgroups of dilatations and translations are the sets $\left\{(a, 0) ; a \epsilon \mathrm{G}_{0}\right\}$ and $\{(1, \gamma) ; \gamma \epsilon \mathrm{K}\}$. In the following we shall sometimes write simply $a$ and $\alpha$ instead of $(a, 0)$ and $(1, \gamma)$ respectively.

'In the course of the proof we make use of the lemma 5.2.3. of (3), the proof of which has not been published yet.
} 
$G_{0}$ acts ergodically on $X$. For $c \in G_{0}$ we have $(c, 0)^{-1}(a, \alpha)=\left(c^{-1} a, c^{-1} \alpha\right)$, so that

$$
\begin{aligned}
\left(\bar{U}_{c} F\right)(a, \chi)=\sum_{\alpha \in K^{+}} f\left(c^{-1} a, c^{-1} \alpha\right) & \alpha(\chi) \\
& =\sum_{\alpha \in K^{+}} f\left(c^{-1} a, \alpha\right)(c \alpha)(\chi)=F\left(c^{-1} a, \chi^{c}\right)
\end{aligned}
$$

so that the operator $\bar{U}_{c}$ in $H$ corresponding to $U_{c}$ is defined for $F \in H$ by $\left(\bar{U}_{c} F\right)(a, \chi)=F\left(c^{-1} a, \chi^{c}\right)$. For $\gamma \in K^{+}$

$$
(1, \gamma)^{-1}(a, \alpha)=(a, \alpha-\gamma) \text {. }
$$

Introducing for a bounded measurable function $\phi(\chi)$ on $X$ the operator $L_{\phi}$ by $\left(L_{\phi} F\right)(a, \chi)=\phi(\chi) F(a, \chi)(F \in H)$, we get easily that the operator corresponding to $V_{\alpha}\left(\alpha \in K^{+}\right)$in $H$ is $L_{\alpha(\chi)}$. Since the operators $U_{a}$ and $U_{\alpha}\left(a \in G_{0}\right.$, $\left.\alpha \in K^{+}\right)$generate $\mathbf{M}(G)$, its image in $H$ is generated by the operators $\bar{U}_{a}$ and $L_{\alpha(\chi)}$. By virtue of the completeness of the system of characters $\{\alpha(\chi)\}$, this ring is identical with the ring generated by the operators $U_{a}$ and $L_{\phi}$, where $\phi(\chi)$ is an arbitrary bounded measurable function on $X$. But, according to a result of Murray and Neumann, operator rings represented in this form are $\mathrm{II}_{1}$ factors of approximately finite type (3, p. 787, Lemma 5.2.3).

Lemma 3. The ring $\mathbf{P}\left(G_{0}\right)$ is a maximal singular abelian subring of $\mathbf{M}(G)$.

Proof. (cf. (1 p. 282, 11. 22-36)).

(a) We have $(g, 0)(a, \alpha)(g, 0)^{-1}=(g, 0)\left(a g^{-1}, \alpha\right)=(a, g \alpha)$. Hence if $\alpha \neq 0$, varying $g$ over $G_{0}$ we get infinitely many different elements of the group $G$.

(b) To prove that $\mathbf{P}\left(G_{0}\right)$ is singular, let $B=\left\{\left(a_{j} ; \alpha_{j}\right)=\bar{a}_{\jmath}, j=1,2, \ldots, n\right\}$ be a finite subset of $G$, and $\bar{a}=(a, \alpha) \bar{\epsilon} G_{0}$. For $g \in G_{0}$ we have

$$
\begin{aligned}
\bar{a}_{i g} \bar{a}_{j}^{-1}=\left(a_{i}, \alpha_{i}\right)(g, 0)\left(a_{j}, \alpha_{j}\right)^{-1}=\left(a_{i}, \alpha_{i}\right)(g, 0)\left(a_{j}^{-1},-\alpha_{j} / a_{j}\right) \\
=\left(a_{i}, \alpha_{i}\right)\left(g a_{j}^{-1},-g \alpha_{j} / a_{j}\right)=\left(a_{i} g a_{j}^{-1},-g \alpha_{j} a_{i} / a_{j}+\alpha_{i}\right)
\end{aligned}
$$

and $\bar{a} g \bar{a}^{-1}=(g, \alpha-g \alpha)$. Since $\alpha \neq 0$, this element is not in $G_{0}$ for $g \neq 1$. $\bar{a}_{i} g \bar{a}_{j}^{-1}=g$ implies $a_{i}=a_{j}$, and $-g \alpha_{j}+\alpha_{i}=0$, so that if in addition for every $i, j$ and $\alpha_{j} \neq 0, g \neq \alpha_{i} / \alpha_{j}$, we have $\bar{a}_{i}=\bar{a}_{j}$.

Now we are going to find out more about the structure of the $\operatorname{ring} \mathbf{R}\left(\mathbf{P}\left(G_{0}\right)\right)$ (in the following denoted simply by $\mathbf{R}$ ), by specializing the group $G_{0}$ appropriately. We know (cf. above), that $\mathbf{R}$ is generated by the set of operators $\left\{U_{g}, V_{h} ; g, h \in G_{0}\right\}$.

Lemma 4. Let $n+1$ be the number of double cosets of $G$ according to the subgroup $G_{0}(n=1,2, \ldots,+\infty)$. Then $R$ is the direct sum of a maximal abelian ring with an abelian ring of uniform multiplicity $n$.

Proof. Let $\Gamma$ be the set of double cosets of $G$ according to $G_{0}$, which differ from $G_{0}$. For $\gamma \in \Gamma$ let us put 


$$
\begin{aligned}
& \mathfrak{M}_{\gamma}=\left\{f(x) ; f(x) \in L^{2}(G) ; f(x)=0, x \bar{\epsilon} \gamma\right\} \\
& \mathfrak{M}_{0}=\left\{f(x) ; f(x)=0, x \bar{\epsilon} G_{0}\right\} .
\end{aligned}
$$

Then $L^{2}(G)$ is the direct sum of the mutually orthogonal subspaces $\mathfrak{M}_{0}$ and $\mathfrak{M}_{\gamma}(\gamma \in \Gamma)$. Evidently these subspaces are invariant with respect to the operators $U_{a}, V_{b}\left(a, b \in G_{0}\right)$ and so they all reduce the ring $\mathbf{R}$. Let $a(\gamma)=$ $\left(a_{\gamma}, \alpha_{\gamma}\right)$ be an arbitrary element from $\gamma$. Since $\alpha_{\gamma} \neq 0$, for $g, g^{\prime} \in G_{0} g a(\gamma) g^{\prime}$ $=a(\gamma)$, or $\left(g g^{\prime} a_{\gamma}, \alpha_{\gamma} g\right)=\left(a_{\gamma}, \alpha_{\gamma}\right)$ implies $g^{\prime}=g=1$. Therefore, if for $f \in \mathfrak{M}_{\gamma}$ we define the function $f^{\prime}$ on $G_{0} \times G_{0}$ by $f^{\prime}(x, y)=f(x a(\gamma) y)\left(x, y \in G_{0}\right)$, we get an isometric mapping between $\mathfrak{M}_{\gamma}$ and $L^{2}\left(G_{0} \times G_{0}\right)$ such that to the operators $U_{a}$ and $V_{b}$ correspond translations by the same elements in $L^{2}\left(G_{0} \times G_{0}\right)$ acting on $x$ and $y$ respectively. In particular, for $\gamma, \gamma^{\prime} \in \Gamma$ there exists an isometric mapping between the spaces $\mathfrak{M}_{\gamma}$ and $\mathfrak{M}_{\gamma^{\prime}}$ such that the restrictions of the operators $U_{a}$ and $V_{b}$ in these subspaces correspond to each other under this mapping. From this it follows at once that the restriction of the ring $\mathbf{R}$ in the orthogonal complement of the subspace $\mathfrak{M}_{0}$ is the $n$-fold copy of its restriction to any of the subspaces $\mathfrak{M}_{\gamma}$.

Similarly $\mathfrak{M}_{0}$ can be identified with the space $L^{2}\left(G_{0}\right)$.

Let $X$ be the character group of $G_{0}$. Then $X \times X$ is the character group of $G_{0} \times G_{0}$, and via Fourier transforms we have an isometry between the space $L^{2}\left(G_{0} \times G_{0}\right)$ and the Hilbert space of complex-valued functions $f(\phi, \psi)(\phi, \psi \in X)$ square-integrable with respect to the Haar measure $\nu$ on $X \times X$. To the operators $U_{a}$ and $V_{a}$ correspond the multiplications by $a(\phi)$ and $b(\bar{\psi})$. Since these operators generate the ring of operators consisting of multiplication by any bounded measurable function on $X \times X$, we see that the restriction of $\mathbf{R}$ in $\mathfrak{M}_{\gamma}(\gamma \in \Gamma)$ is maximal. Analogous reasoning shows that the restriction of $\mathbf{R}$ in $\mathfrak{M}_{0}$ is maximal abelian too. In order to prove Lemma 4 it evidently suffices to show that the restriction of $\mathbf{R}$ in the space $\mathfrak{M}_{0} \otimes \mathfrak{M}_{\gamma}(\gamma$ arbitrarily chosen from $\Gamma$ ) contains the projection on the subspace $\mathfrak{W}_{0}$. By reasonings applied above this amounts to the following: Let $Z$ be the sum of the topological spaces $X$ and $X \times X$, and let $L^{2}(Z)$ be the Hilbert space of functions on $Z$ square-integrable with respect to a measure $\tau$, which coincides on $X$ and $X \times X$ with the Haar measures of these compact topological groups respectively. For $a, b \in G_{0}$ and $F(p) \in L^{2}(X)(p \in Z)$ let us define

$$
\left(L_{a, b} F\right)(p)=h_{a, b}(p) F(p)
$$

where

$$
h_{a, b}(p)=\left\{\begin{array}{lll}
a(\chi) \overline{b(\chi)} & \text { if } & p=\chi \in X \\
a(\phi) \overline{b(\psi)} & \text { if } & p=(\phi, \psi) \in X \times X .
\end{array}\right.
$$

Then all that we have to prove is that the ring generated by the operators $L_{a, b}$ contains the multiplication by the characteristic function of $X$. If a sequence of linear combinations of the functions $h_{a, b}(p)\left(a, b \in G_{0}\right)$ converges 
on $X \times X$ uniformly towards a continuous function, then it converges uniformly on $Z$ towards a continuous function $f(p)$ which satisfies $f\left(p_{1}\right)=f\left(p_{2}\right)$ if $p_{1}=\chi \in X$ and $p_{2}=(\chi, \chi) \in X \times X$. Conversely, every continuous function on $Z$ satisfying this condition can be obtained in this way. Therefore the ring generated by the operators $L_{a, b}$ contains multiplications by such functions. But since the characteristic function of the set $X \subset Z$ can be obtained as limit of a bounded sequence of them, converging almost everywhere with respect to the measure $\tau$, our lemma is proved.

In order to obtain a sequence of singular maximal abelian subrings with the desired properties in a $\mathrm{II}_{1}$ factor of approximately finite type, by virtue of Lemmas 2, 3, and 4 it suffices to prove the following.

Lemma 5. Let $n$ be a positive integer. Then there exists a field $K$ which is the union of an increasing sequence of finite subfields, a subgroup $G_{0}$ of the multiplicative group $K^{*}$ of $K$, such that if $G$ is the subgroup of the affine group over $K$ corresponding to $G_{0}$, the number of double cosets of $G$ according to $G_{0}$ equals $n+1$.

Proof. We shall perform this in two steps.

(a) Let $G_{0}$ be a subgroup of the multiplicative group of $K$, which has the index $n$. We show that the number of double cosets of $G$ according to $G_{0}$ is $n+1$. To see this, we observe, that if $\bar{a}=(a, \alpha)$ and $\vec{b}=(b, \beta)$ are in the same double coset then $g \bar{a} g^{\prime}=b$, or $\left(g g^{\prime} a, g \alpha\right)=(b, \beta)$, and so $\beta=g \alpha$ $\left(g, g^{\prime} \in G_{0}\right)$. The converse can be proved similarly.

(b) ${ }^{7}$ According to (a) it suffices to find a $K$ and a $G_{0}$, such that $G_{0}$ has the index $n$ in $K^{*}$. Let $p$ be a prime number, such that $n$ is a divisor of $p-1$; let $n^{s}$ be the greatest power of $n$ which divides $p-1$. For an integer $k>0$, let us denote by $F_{k}$ the field of order $p^{k}$, and by $F_{k}$ the multiplicative group of $F_{k}$. Let $k_{1}<k_{2}<$ be a sequence of integers relatively prime to $n$. We have

$$
F_{k_{1}} \subset F_{k_{2}} \subset \ldots
$$

We denote by $K$ the union of the fields

$$
F_{k i}(i=1,2, \ldots,) \text {. }
$$

We are going to show that $K$ is the direct product of a subgroup with a cyclic group of order $n^{s}$, from which the existence of the $G_{0}$ with the required properties follows at once. The number of elements of $F_{k}$ is

$$
p^{k}-1=(p-1)\left(p^{k-1}+p^{k-2}+\ldots+1\right) .
$$

On the other hand,

$$
p^{k_{i-1}}+p^{k_{i}-2}+\ldots+1 \equiv k_{i}(\bmod n) .
$$

\footnotetext{
${ }^{7}$ The author is indebted to the referee for this part of the lemma which makes it possible to avoid the use of Lemma 2 and hence that of 5.2.3 in (3).
} 
So $n^{s}$ is the highest power of $n$ dividing $p^{k_{i}}-1$. Since $F^{\times}$is a cyclic group, it contains a cyclic group $H$ of order $n^{s}$, which is a direct factor, and which does not change with $i$. So $H$ is a direct factor in $K^{\times}$, which proves our lemma. We sum up the preceding lemmas in the following theorem:

THEOREM. If $M$ is an approximately finite factor of Type $\mathrm{II}_{1}$ then it contains an infinite sequence of singular maximal abelian subrings which cannot be pairwise connected by *automorphisms of $M$.

\section{REFERENCES}

1. J. Dixmier, Sous-anneaux abéliens maximaux dans les facteurs de type fini, Ann. Math., 5:9 (1957), 279-286.

2. F. J. Murray and J. von Neumann, On rings of operators, Ann. Math., 37 (1936), 116-229. 3. - On rings of operators IV, Ann. Math., 44 (1943), 716-808.

4. I. E. Segal, Decompositions of operator algebras II, Mem. Amer. Math. Society, no. 9 (1951).

RIAS, Baltimore 